\title{
ENHANCEMENT OF SOLUBILITY AND DISSOLUTION RATE OF CURCUMIN USING POROUS STARCHBY SOLID DISPERSION TECHNIQUE
}

\author{
D.O.I - 10.51201/Jusst12631 \\ http://doi.org/10.51201/Jusst12631
}

\author{
Nikita D. Gidde*, Priyanka V. Desai, Priyanka V. Bagade \\ MayuriS. Lokhande, Indrayani D.Raut, Manojkumar M. Nitalikar \\ Rajarambapu College of Pharmacy, Kasegaon (MS) India
}

\begin{abstract}
The poor dissolution characteristics of biopharmaceutical class II drugs are a major concern for scientists in thepharmaceutical industry. Solid dispersion is introduced as a novel method for enhancement of solubility. Class IIdrugs are low solubility and high permeability according to the biopharmaceutical classification system and arepromising candidates for improving solubility and bioavailability through solid dispersion. The purpose of the present attempt is to prepare a soliddispersion of curcumin and porous starch in order to increase the solubility and dissolution of drugs that are poorlysoluble. Solid dispersions (SDs) of BCS-II drugs were prepared by ball milling in ratio of drug:polymer i.e.curcumin:porous starch (1:0.5, 1:1, 1:2 and 1:3). Further, SDs were investigated by solubility, FTIR, XRD, DSC,micromeritics, and in-vitro dissolution. . Conclusively, porous starch offers a hydrophilic matrix to deliver poorwater soluble drugs and Solid dispersion system have demonstrated an improved performance. Solid dispersionsystem have demonstrated an improved performance
\end{abstract}

Key wards: Solid dispersion, solubility, bioavailability, BCS II drugs.

\section{INTRODUCTION}

A drug's oral bioavailability depends on its solubility and/or dissolution rate, and dissolution can be a rate determining step for the appearance of a medicinal effect, so efforts are often required to increase the dissolution of a drug with limited water solubility. Several methods, 
including salt formation, micro ionization, and solvent or surface-active agent addition, are available to improve these characteristics. Strong dispersion (SD) is one of these processes, involving the dispersion of one or more active ingredients into an internal carrier or strongstate matrix prepared by melting, solvent dissolution or melting solvent process [1].

GI tract drug absorption can be restricted by a number of factors, including poor aqueous solubility and poor membrane permeability of the drug molecule, which are the most important contributors. If an active agent is administered orally it must first dissolve in gastric and/or intestinal fluids before it can penetrate the GIT membranes to enter systemic circulation. Therefore, there are two areas of pharmaceutical research focused on enhancing the oral bioavailability of active agents: increasing the solubility and dissolution rates of poorly soluble water products. The BCS is a theoretical method focused on its aqueous solubility and intestinal permeability for classifying a drug product.As for BCS class II \& IV drug rate limiting step is drug release from the dosage form and solubility in gastric fluid and not absorption, so increasing the solubility in turn increases the bioavailability for BCS class II \& IV drugs [2,3].

Curcumin is a naturally occurring compound of curcuma longa L. In both preclinical and clinical trials, and has demonstrated several pharmacological activities such as antiinflammatory, antioxidant. Moreover, curcumin has hepatoprotective, neuroprotective function and protective against myocardial infraction. In particular, curcumin has also shown favorite anticancer efficacy, but limiting factors such as its extremely low oral bioavailability hamper its use as a therapeutic agent. It is essentially insoluble in water, and is particularly susceptible to changes in $\mathrm{pH}$. Chemically, curcumin is a bis-Unsaturated diketone that exhibits keto-enol tautomerism in acidic and neutral solutions with a predominant keto form and stable alkaline enol form. Approximately 77 percent diffuloylmethane, 17 percent demethoxycurcumin and 6 percent bisdemethoxycurcumin are present in commercial curcumin.Low intrinsic activity, low absorption, high rate metabolism, inactivity of metabolic products and/or rapid removal and clearance from the body are the reasons for any agent's decreased bioavailability within the body. To date, studies have indicated a high intrinsic activity and, thus, curcumin's usefulness as a therapeutic agent for various ailments. However, studies relating to the absorption, distribution, metabolism and excretion of curcumin over the past three decades have shown poor absorption and rapid metabolism of curcumin, which severely restricts its bioavailability [4].

Recently, a growing number of reports have been published detailing biomaterials extracted from food sources, which display significant benefits in different applications. 
Due to their abundant supply, renewability and relatively low cost, natural biopolymers are the most commonly used. The application of starch as adsorbents, however, is constrained by its natural surface areas and volumes of pore. To overcome these deficiencies, different modified starch was produced to obtain high surface area and pore volumes. The material that has received significant attention in recent years is porous starch. Porous starch is a new modified starch that stretches to the center with abundant micronized pores from the surface of starch granules. The diameter of the pores is about $1 \mathrm{~m}$, and about half of the starch is the total volume of the pores. Porous starch is obtainable by physical, chemical, and biological processes. A simple and green method for preparing porous corn starch based on amylase and glucoamylase used as hydrolysis enzymes has been introduced in the research. Porous starch has excellent adsorption properties as compared with native starch or other modified starch due to its large specific surface area [5].

Curcumin has low bioavailability and is virtually insoluble in water. The solubility depends on many of the essential pharmaceutical properties and processes such as dissolution rate, moiety absorption, distribution and excretion. Approximately 40 percent of orally administered medications suffer from low water solubility-related training difficulties. In this work, we investigate the solubility of curcumin, thus enhancing the solubility and bioavailability of curcumin. Solid dispersion Hydrophilic polymer preparation also faces problems with softness and tackiness. The use of large quantities of polymer is necessary to solve such problems. Using higher quantities of polymer also results in large dosage type tablet weight which is basically unacceptable. Therefore, the preparation of porous starch was thought to solve problems of formulation associated with solid dispersions. In addition, through geometrical constraints, the porous structure is able to effectively suppress the crystallization of amorphous material, as well as due to changes in nucleation mechanisms and kinetics within the small pores and the interaction between API and the pore wall, which can cause a transformation of the crystalline to amorphous phase [1].

\section{MATERIALS AND METHODS}

\subsection{Materials}

Curcumin and alcohol were obtained as gift sample from Research Lab Fine Chem Industry. Starch (Corn) and liquid paraffine was procured from S.D Lab Chemicals. Potassium dihydrogen Phosphate was purchased from LOBAChemieIndoAustranal.Sodium Hydroxide was obtained from Merck Life Science.All other solvents and reagents were of analytical grade. 


\subsection{Authentication of Pure Curcumin}

\section{a) Organoleptic characteristics}

Organoleptic characteristics are those characteristics that are evaluated after an impression of the sense organs. It refers to drug evaluation by properties such as - color, odor, taste etc. It is the qualitative evaluation methodology focused on the analysis of drug morphology and sensory profile.

$>$ Color:Color is typically a function of the intrinsic chemical structure of drugs compared to a certain degree of unsaturation. While structurally saturated, some compounds can appear to have color.

$>$ Odor:The substance can present an inherent odor characteristic of important functional classes.

$>$ Taste:If taste is considered unpalatable, the use of a less soluble type of a drug should be taken into account [6].

\section{b) Determination of Meting point}

The procedure used to calculate the melting point of curcumin was capillary fusion. An appropriate quantity of drug was filled into the capillary in this one-sided, sealed capillary. The capillary was held within the apparatus of the melting point, and temperature gradually increased. The temperature was noted down with the aid of a thermometer when the drug began to melt and when completely melted [6].

\section{c) Solubility studies}

The determination of the maximum solid mass that can be dissolved at a given temperature in $100 \mathrm{ml}$ of solvent is one way of calculating solubility. The drug's solubility reflects the drug's bioavailability. Intrinsic solubility, $\mathrm{pH}$ solubility profiling, pharmaceutical solvent solubility, and surfactant effects are used in solubility studies.Solubility research focuses on drug solvent mechanism that could occur during drug candidate delivery [6].

\section{d) Determination of Calibration curve}

Using UV -visible spectrophotometer, the maximum absorption of curcumin in phosphate buffer $\mathrm{pH} 7.4$ was measured by scanning the drug solution within the range of 400-800 $\mathrm{nm}$. The drug was found to demonstrate maximum absorption at $432 \mathrm{~nm}$. 


\section{$>$ Preparation of stoke solution}

Weighed $100 \mathrm{mg}$ of curcumin and solubilized in $5 \mathrm{ml}$ of methanol which dissolved further up to $100 \mathrm{ml}$. Working standard was prepared with phosphate buffer $7.4(\mathrm{pH})$ to achieve a concentration of $1 \mathrm{mg} / \mathrm{ml}$ using this stock solution.

\section{$>$ Preparation of working standard-}

$10 \mathrm{ml}$ of stock solution was taken, and the phosphate $\mathrm{pH} 7.4$ volume was used to achieve 100ug / ml concentration. Further dilutions were made to give curcumin concentration of 5,10,15,20,25and 30ug / $\mathrm{ml}$, respectively. This solution was scanned and the absorbance was measured at $432 \mathrm{~nm}$. The resulting absorption value was plotted against the respective concentration to obtain the standardized curve[6].

\subsection{Preparation of porous starch}

Ten gramsof starch had been well distributed at room temperature in $40 \mathrm{~mL}$ of distilled water. Sixty milliliters of distilled water up to its boiling point was heated. Starch dispersion was then applied under quick stirring to the boiling water. The temperature was brought down steadily to room temperature, and stirring continued until a translucent gel was formed.The gel was held overnight in excess water at $8^{\circ} \mathrm{C}$ to reach an equilibrium state. The gel was then exposed to ethanol for solvent exchange. In order to retain its porous shape, the gel was stabilized and stored in ethanol at $8^{\circ} \mathrm{C}$ for $48 \mathrm{~h}$. After the equilibrium state was reached, the gel was then dried under a vacuum at $30^{\circ} \mathrm{C}$ using rotary vaporization. The dried material was milled in the vacuum and processed for further use. In addition, this substance is referred to as Porous starch [5].

\subsection{Preparation of solid dispersions}

Curcumin was prepared with solid dispersions (SDs) by spray drying, solvent evaporation, ball milling and physical mixing techniques. Using the ball milling method, batches of solid dispersions for curcumin were prepared by varying the drug ratio: Porous Starch (1:0.5, 1:1, $1: 2$, and 1:3 w/ w).The procedure followed for the respective method is defined as follows for the preparation of Curcumin drug ball milled SDs with porous starch were subjected to ball friction at $100 \mathrm{rpm}$ for $3 \mathrm{~h} \mathrm{[7].}$ 


\begin{tabular}{|c|c|c|}
\hline Ratio & Curcumin (mg) & Porous Starch(gm) \\
\hline $1: 0.5$ & 500 & 0.250 \\
\hline $1: 1$ & 500 & 0.500 \\
\hline $1: 2$ & 500 & 1 \\
\hline $1: 3$ & 500 & 1.5 \\
\hline
\end{tabular}

Table No:1: Different Ratios of Curcumin Solid Dispersion using Porous Starch

\subsection{Micromeritics study}

In terms of angle of repose, Carr index and Hausner ratio, the flow properties of the drug and solid dispersion have been defined. The sample was poured through the walls of a funnel to determine the angle of repose, which was set in a position such that its lower tip was precisely $2.0 \mathrm{~cm}$ above the hard surface at its height. The sample was poured until the lower tip of the funnel was touched by the upper tip of the surface of the pile. The angle of repose was given by Thetan-1 (height of the pile / radius of its base). The sample was gently poured into a graduated cylinder with a glass funnel cut exactly to $10 \mathrm{ml}$ mark.Using a spatula, the excess sample was extracted and the weight of the cylinder with the powder needed for cylinder volume filling was measured. Until there was no further volume drop, the cylinder was then tapped from a height of $2.0 \mathrm{~cm}$. The bulk density $(\rho b)$ and tapped density $(\rho t)$ were assessed.

Hausner ratio (HR) and Carr index (CI) were calculated according to the two equations given below:[8]

$$
\begin{aligned}
& H R=\rho t / \rho b \\
& C I=(\rho t-\rho b) / \rho t \times 100
\end{aligned}
$$

\subsection{Phase Solubility Study}

The drug and solid dispersion must be present at the absorption site in the form of a solution for all mechanisms of absorption. During the preformulation analysis the drug's solubility must be determined. Drug solubility tests were carried out on the basis of the method described by Higuchi and Connors. An excess of curcumin was put separately in $25 \mathrm{ml}$ of screw-capped glass vial capacity containing $20 \mathrm{ml}$ of Water and Phosphate Buffer solution. The samples were shaken in orbital shaker for $48 \mathrm{~h}$ at $37 \pm 0.5 \mathrm{C}$ (100 agitations / $\mathrm{min})$. The solutions were filtered through a filter membrane after obtaining equilibrium (pore size 0.45 
m).The filtered samples have been properly diluted and U.V. Take Absorbance Spectrophotometer at $421 \mathrm{~nm}$ [7].

\subsection{In Vitro Dissolution Study}

Dissolution tests of all SDs (equivalent to $10 \mathrm{mg}$ of drug) were conducted using a dissolution test apparatus of USP Pharmacopeia type II The dissolution test condition was as follows: medium-900 ml phosphate buffer (pH7.4); pace-100 rpm; temperature $37 \pm 0.5 \mathrm{C} .5 \mathrm{ml}$ of aliquot was removed at various time intervals from 5 to $120 \mathrm{~min}$ during the dissolution analysis and replaced with an equal amount of fresh medium. The samples removed were filtered and absorbance measured at $421 \mathrm{~nm}$, respectively [7].

\subsection{FTIR Spectroscopy}

In this analysis, the potassium bromide disc method was applied to the spectrophotometer. Drug and Solid dispersions had been applied for an IR analysis. IR grade potassium bromide was closely mixed with the powdered sample. Using special dies, the mixture was then compacted into transparent disc under high pressure. The disc was put with the sample holder in the IR spectrophotometer and the spectrum was registered [1].

\subsection{Powder X-ray Diffraction (XRD)}

The distinctive X-ray diffractogram is provided by crystalline compounds. This diffraction pattern is useful when distinguishing compounds. A calculation of a sequence of $d$ spacings, the interplanar spacings from the direction of the diffraction peaks, is a quantitative study of the $\mathrm{X}$ ray powder diffraction technique.During the data collection the sample was rotated to minimize orientation effects, and the data was collected using a curved photosensitive detector [1].

\subsection{Differential Scanning Calorimetry (DSC)}

Drug and solid Dispersion thermal behavior was analyzed using Calorimetry differential scanning instrument fitted with a Stare computer programmer. Using differential calorimeter scanning, DSC thermograms were taken. Around 2-5 mg of the sample was heated under a nitrogen stream in an open aluminum pan from $30-300^{\circ} \mathrm{C}$ at a scanning rate of $10^{\circ} \mathrm{C} / \mathrm{min}$ [1]. 


\section{RESULT AND DISCUSSION}

\subsection{Authentication of curcumin}

a) Organoleptic Properties

Qualitative assessment focused on the analysis of the drugs' morphological and sensory profile. Organoleptic properties have been measured and the result is shown in table2

\begin{tabular}{|c|c|c|}
\hline Color & Odor & Taste \\
\hline Yellow & Earthy & Bitter \\
\hline
\end{tabular}

Table No. 2: Organoleptic Properties of curcumin

b) Melting Point

The drug's melting point was observed using the technique of capillary fusion. The drug's measured melting point has been found to be in the reference value range. The findings are shown in Table 3.1.b

\begin{tabular}{|c|c|c|}
\hline Apparatus & Observed value & Reference Value \\
\hline Melting Point Apparatus & $182^{0}-184^{\circ} \mathrm{C}$ & $179^{0}-183^{0} \mathrm{C}$ \\
\hline
\end{tabular}

\section{Table No. 3: Melting Point of Curcumin}

c) Solubility Study

Solubility of the drug represents the bioavailability of the drug. The results are shown in table 3.1.c

\begin{tabular}{|c|c|}
\hline Insoluble & cold water, ether \\
\hline Soluble & Alcohol, Glacial acetic acid \\
\hline Very Soluble & Ethanol \\
\hline
\end{tabular}

Table No. 4: Solubility study of Curcumin

\section{d) Calibration Curve}

The curcuminin phosphate buffer (7.4) calibration curve using UV-Spectrometer 432 $\mathrm{nm}$ was performed and the result is shown in table 3.1.d.and absorption values are in fig 3.1.d 


\begin{tabular}{|c|c|c|}
\hline Sr. No. & Concentration $(\mathbf{u g} / \mathbf{m l})$ & Absorbance \\
\hline 1 & 5 & 0.081 \\
\hline 2 & 10 & 0.145 \\
\hline 3 & 15 & 0.212 \\
\hline 4 & 20 & 0.277 \\
\hline 5 & 25 & 0.337 \\
\hline 6 & 30 & 0.415 \\
\hline
\end{tabular}

Table No. 5 : Observation Table curve of Curcumin

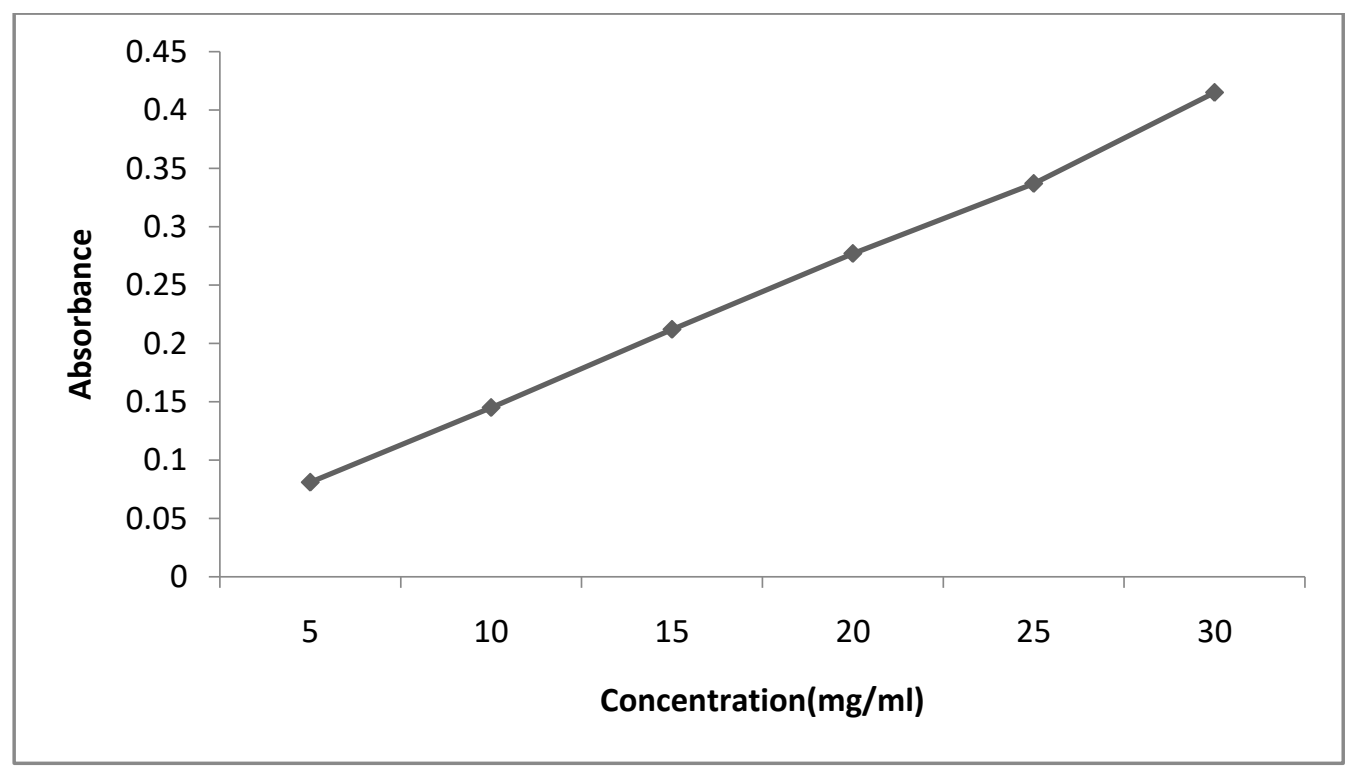

Figure No 1: Curve of Curcumin in phosphate buffer (7.4) by UV- Spectrophotometer at $432 \mathrm{~nm}$

\subsection{Flow Properties}

Further Table shows the angle of rest, bulk density, tapped density, Hausner 's ratio and Carr's index values of formulations. 


\begin{tabular}{|c|c|c|c|c|c|}
\hline $\begin{array}{c}\text { Batch } \\
\text { Code }\end{array}$ & $\begin{array}{c}\text { Angle of } \\
\text { Repose( }\end{array}$ & $\begin{array}{c}\text { Bulk } \\
\text { Density(gm/cc) }\end{array}$ & $\begin{array}{c}\text { Tapped } \\
\text { Density(gm/cc) }\end{array}$ & $\begin{array}{c}\text { Hausner } \\
\text { Ratio }\end{array}$ & $\begin{array}{c}\text { Carr's } \\
\text { Index(\%) }\end{array}$ \\
\hline Curcumin & 37.88 & 0.3488 & 0.3564 & 1.194 & 17.25 \\
\hline F1 & 32.56 & 0.3643 & 0.418 & 1.143 & 13.06 \\
\hline F2 & 33.56 & 0.2753 & 0.316 & 1.147 & 13.5 \\
\hline F3 & 34.89 & 0.334 & 0.385 & 1.145 & 13.32 \\
\hline F4 & 35.25 & 0.3716 & 0.402 & 1.168 & 35.25 \\
\hline
\end{tabular}

Table No.6: Flow properties of Solid Dispersion of Curcumin prepared by using porous Starch

From the above result, solid dispersion indicated good Flowability.

\subsection{Saturated Solubility Study}

Table 3.3.1 demonstrates the saturation solubility of curcumin in distilled water and PBS and its SDs. It can be inferred from the findings that solid dispersion increases the solubility of the drugs in saturation. Thus, porous starch has improved the solubility of curcumin.

\begin{tabular}{|c|c|c|}
\hline \multirow{2}{*}{ Batch Code } & \multicolumn{2}{|c|}{ Saturated Solubility (mg/ml) } \\
\cline { 2 - 3 } & Distilled Water & Phosphate Buffer Solution \\
\hline Curcumin & 0.22 & 0.29 \\
\hline F1 & 0.34 & 0.38 \\
\hline F2 & 0.38 & 0.41 \\
\hline F3 & 0.40 & 0.48 \\
\hline F4 & 0.42 & 0.49 \\
\hline
\end{tabular}

Table No. 7: Saturated Solubility of Optimized Batches of Solid Dispersion (mg/ml) 


\subsection{In vitro drug dissolution study}

In Fig.3.4.1, the dissolution profiles of curcumin and all batches of SDs were seen. At the end of 120 min, batch F1 showed 88 percent dissolution, and batches F2, F3 and F4 showed almost 98 percent dissolution at the end of $120 \mathrm{~min}$, respectively. Simple curcumin, in comparison to the above, displayed a low dissolution profile at the end of 120 minutes, i.e. just 26.25 percent.Bad curcumin dissolution may have been due to poor wettability and/or agglomeration.

As compared to plain products, the dissolution profiles of all SDs were higher. Batch F2 of SDs in mass ratios 1:1 w/ $\mathrm{w}$ showed a dissolution of $\sim 90$ percent at the end of $60 \mathrm{~min}$, except F1 (1:0.5 w / w) showed a dissolution of $~ 90$ percent at the end of 120 min.In addition, no significant dissolution improvement was shown by increasing the concentration of porous starch.

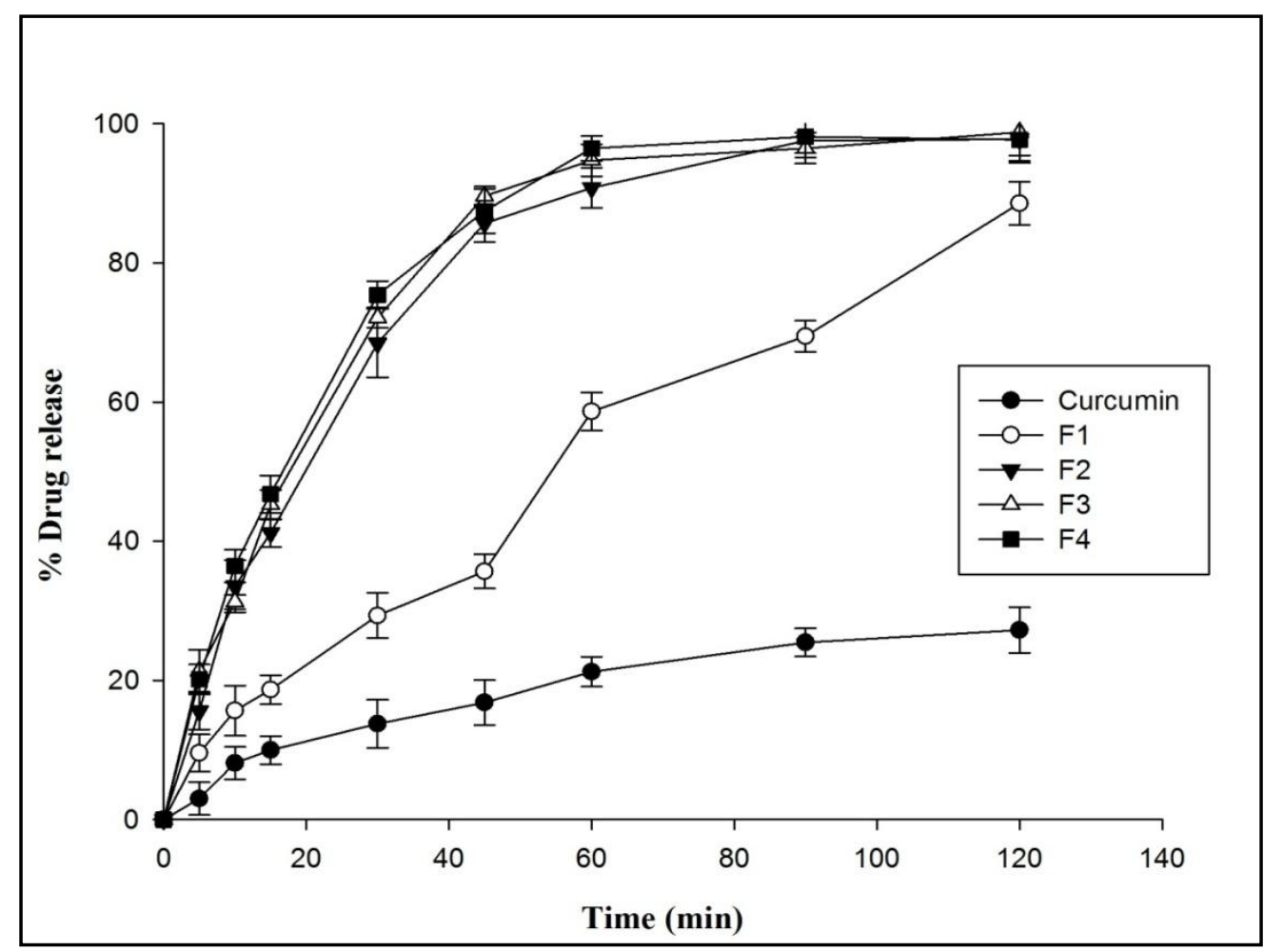

Fig No 2 :In vitro dissolution of curcumin and solid dispersion

The dissolution profile suggests that, as opposed to simple drugs, porous starch shows greater progress in dissolution. This implies that, with porous starch, major changes in dissolution have been observed. The presence of several proton donors and acceptor groups in porous starch has been attributed to the capacity of porous starch to increase the rate of dissolution of poorly soluble drugs. 


\subsection{FTIR spectroscopy}

Curcumin, porous starch and their SDs were seen in the FTIR Spectra in Fig. 3.5.1. The wave number of prominent curcumin peaks showed $3504.66 \mathrm{~cm}-1$ (O-H stretching), $1618.28 \mathrm{~cm}-1$ $(\mathrm{C}=\mathrm{O}$ stretching, ketone) and $1591.27 \mathrm{~cm}-1(\mathrm{C}=\mathrm{C})$ respectively. At their respective locations, porous starch displayed the prominent peaks. The prominent curcumin peaks showed a small change in the continuum of solid dispersion of peaks with reduced strength. It revealed that spectral shifts, such as the slight shift or widening of the curcumin and porous starch peaks, showed a strong degree of interaction between them.

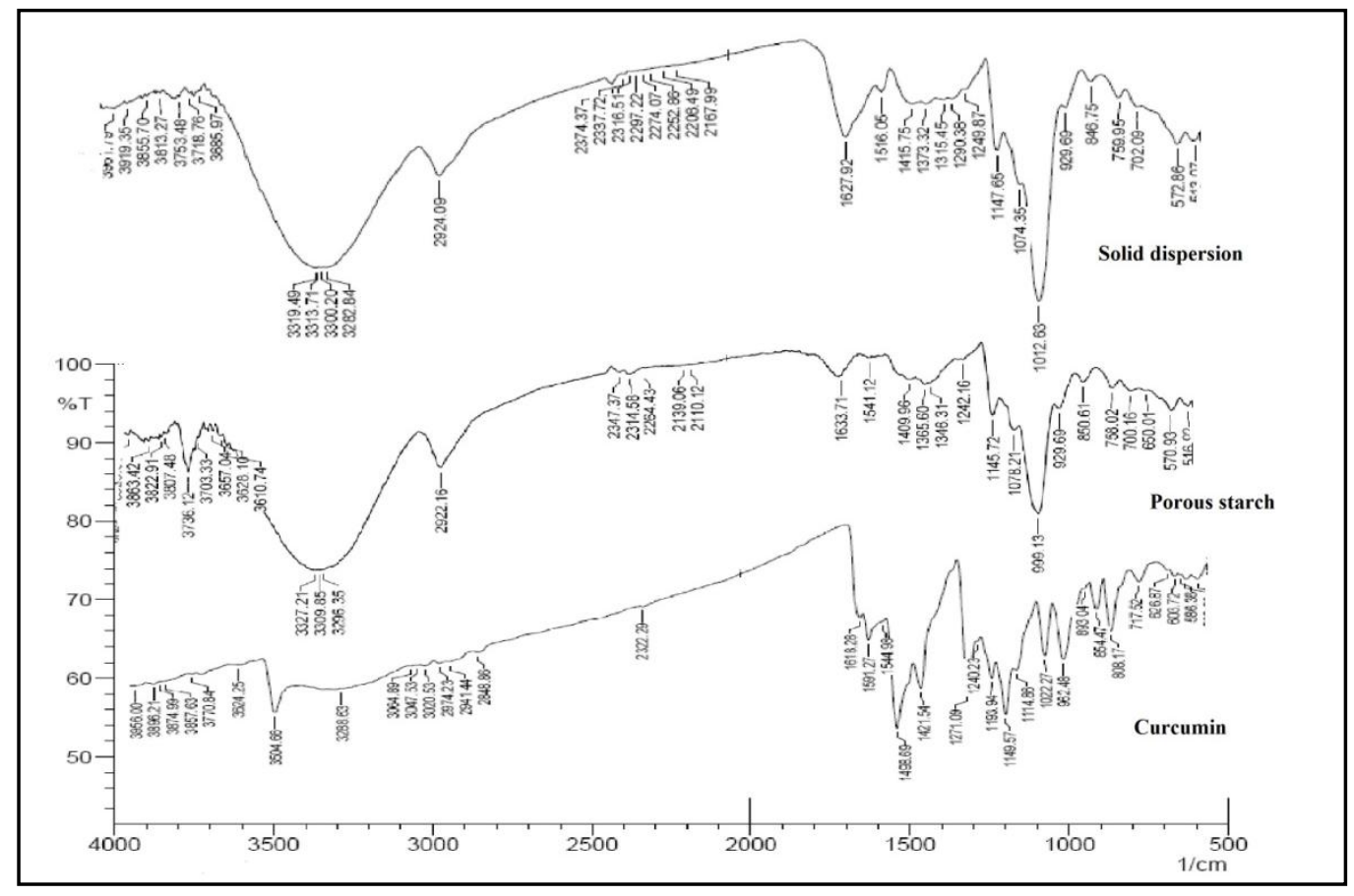

FigNo. 3: FTIRspectra of curcumin, porous starch and solid dispersion

In addition, in the low-frequency region of the spectrum, peaks corresponding to porous starch and curcumin showed only a small change in shifting and widening that could be attributed to intermolecular hydrogen bonding. The broadening of peaks could also be due to the solid dispersion's amorphous shape.

\subsection{Powder X-ray diffraction}

The curcumin's Powder X-ray diffraction patterns showed typical peaks of high-intensity diffraction. The appearance in X-ray diffractogram of numerous sharp, narrow, and intense peaks suggested that the curcumin is highly crystalline in nature. No peaks suggesting its amorphous existence were seen in the X-ray diffraction patterns of the porous starch.The SDs $(1: 1 \mathrm{w} / \mathrm{w})$ showed a large decrease in curcumin-corresponding crystalline peaks, suggesting 
that crystals have been converted to an amorphous or microcrystalline shape. It implies interaction of the solid-state between curcumin and porous starch has been observed.It seems that at molecular level, the degree of dispersion of drugs into porous starch was approaching.

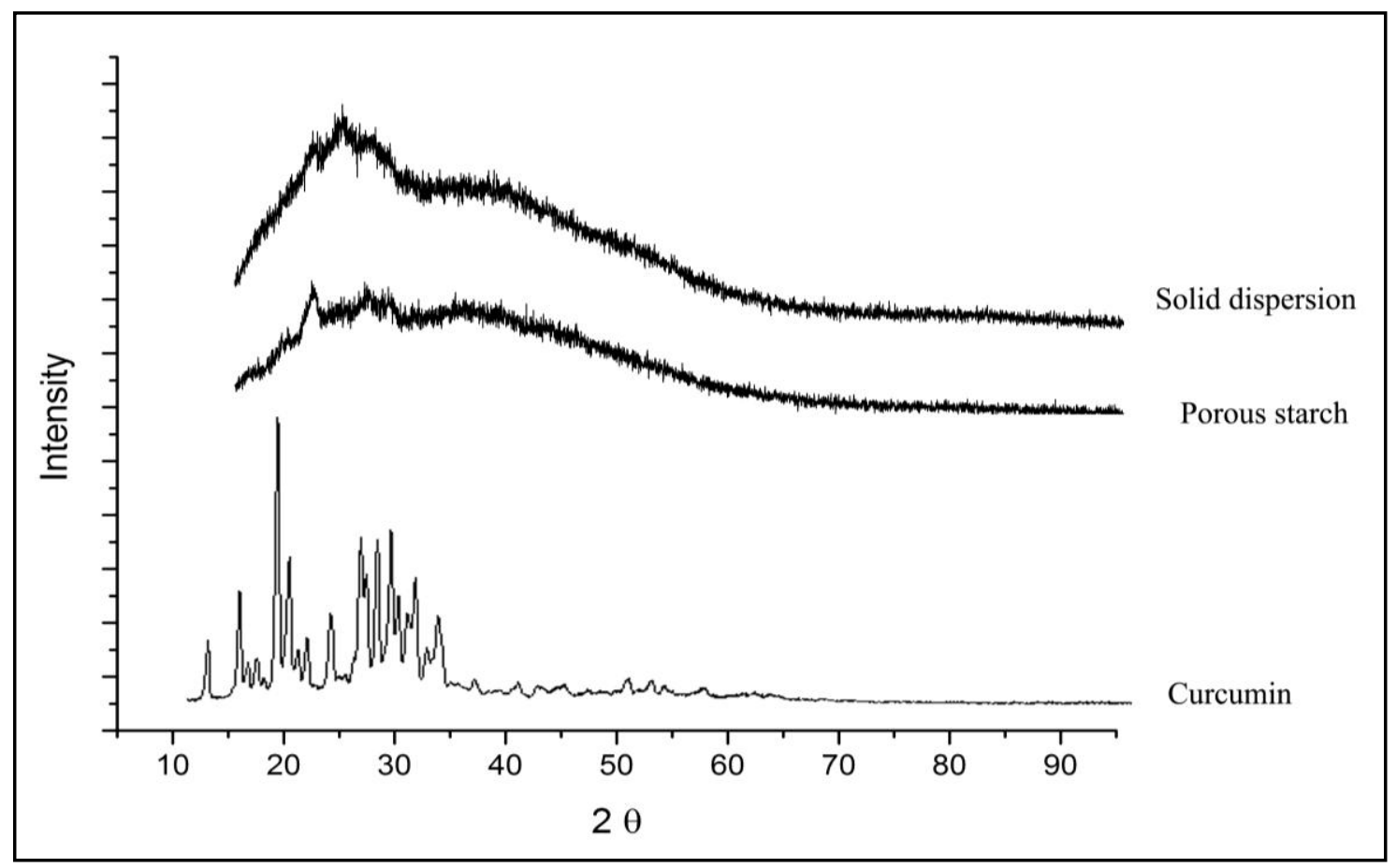

Fig.No. 4: XRD spectra of curcumin, porous starch and solid dispersion

There are polar groups such as, $-\mathrm{OH}$, and - $\mathrm{CO}$ in the molecular structure of curcumin that are capable of interacting with the functional groups present in porous starch, such as hydroxyl, to facilitate interactions with hydrogen, electrostatic and Van der Waals. Consequently, this result indicates that porous starch disrupts and interacts at the molecular level with the crystalline shape of curcumin.

\subsection{Differential scanning calorimetry (DSC)}

The thermograms of curcumin clearly showed sharp endothermic peaks at $173.17{ }^{\circ} \mathrm{C}-180.62$ ${ }^{\circ} \mathrm{C}(\mathrm{H}=-152.66 \mathrm{~J} / \mathrm{g}$ ), (Fig. 3.7.1)From the thermograms of pure curcumin the Sharp narrow peaks were observed, suggesting the drugs' high crystallinity. SDs $(1: 1 \mathrm{w} / \mathrm{w})$ showed a nearly complete reduction of the peak area from the comparison of all thermograms.

The curcumin SDs batches exhibited a large and small endothermic peak corresponding to the melting point. Large endothermal peaks of melting showed that the phase transition and reduction of crystallinity occurred in the dispersions, which may have helped to reduce the drug crystallinity in the dispersions. 


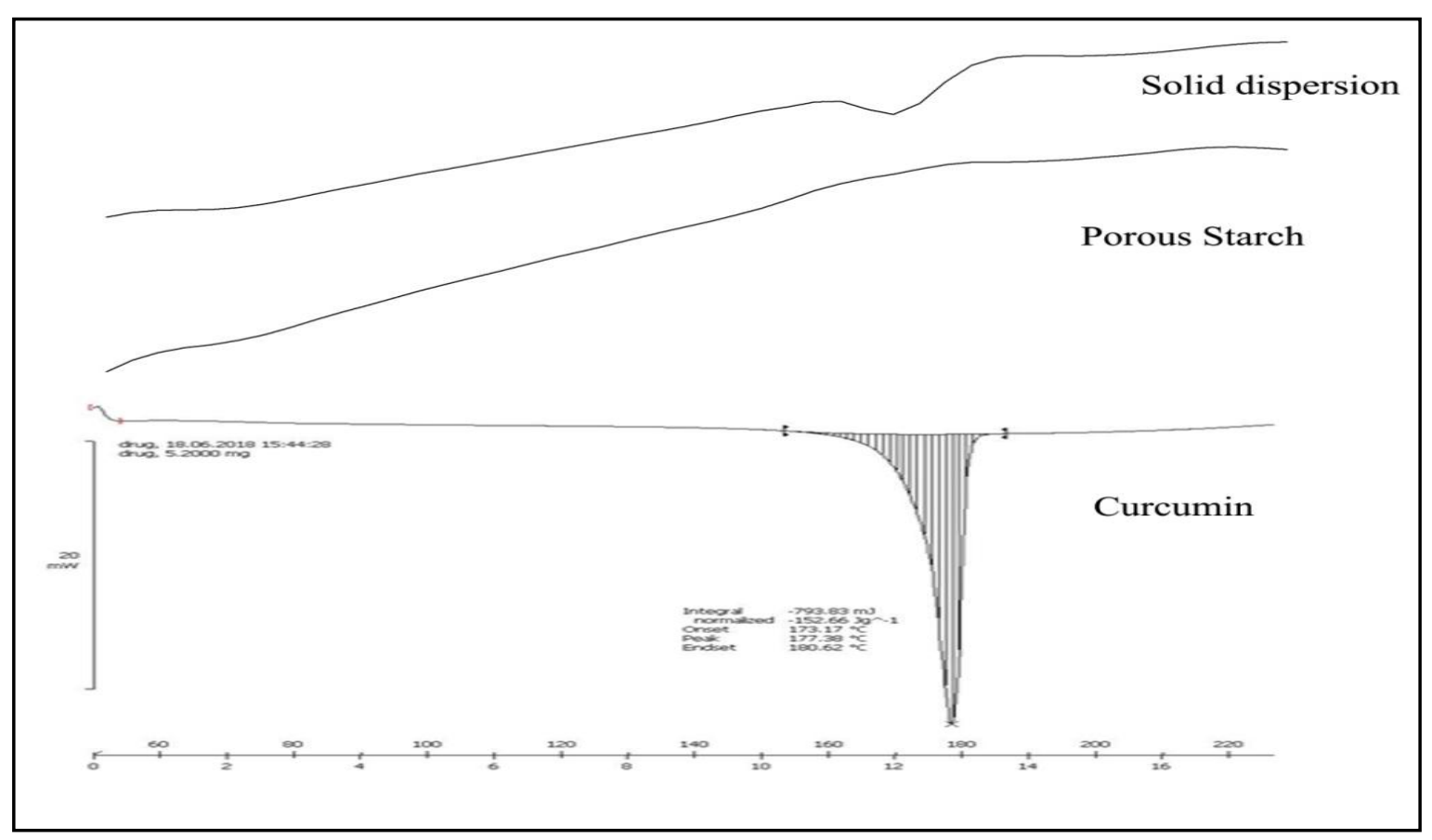

Fig. No. 5: DSC of curcumin, porous starch and solid dispersion

Therefore, the results of the DSC analysis confirm that curcumin is completely transformed into amorphous form and that there have been desirable interactions with porous starch.

\section{CONCLUSION}

The use of porous starch successfully prepared the strong dispersions of curcumin. The solid dispersions formed were characterized in terms of study of solubility, FTIR, study of dissolution, and study of crystallinity (XRD, and DSC).

Curcumin-PS systems have demonstrated an improved performance relative to curcumin. Interestingly, a single fold of porous starch has improved the solubilization of curcumin. Thus, for various other drug candidates, PS may be used as a solubility enhancer and carrier.

\section{REFERENCES}

1. M. K. Modasiya and V. M. Patel Studies on Solubility of Curcumin Int. J. of Pharm.\& Life Sci (IJPLS), Vol.3, Issue 3: March:2012, 1490-1497.

2. DebjitBhowmik, Harish Gopinath, B. Pragati Kumar. S. Duraivel, K. P. Sampath Kumar, Oral Controlled release Drug Delivery System,Vol.1 No. 10 2012,

3. Sandip Kumar, Pritam Singh, Various Techniques for Solubility Enhancement, The Pharma Innovation Journal (2016);5(1):23-28. 
4. PreethaAnand,Ajaikumar B. Kunnumakkara, Robert A. Newman, and Bharat B. Aggarwal, Bioavailability of Curcumin: Problems and Promices, Anand et al., http://pubs.acs.orgon April 2, 2009

5. Meer Tarique Ali, RiteshFule, Ajay Sav, and Purnima Amin, Porous Starch; a Novel Carrier for Solubility Enhancement of Carbamazepine, AAPS Pharma SciTech, Vol.14, No. 3, September 2013 DOI: 10.1208/s12249-013-9985-6

6. NitinSalunkhe, AdhikraoJadhav, NamdeoJadhav, Screening of drug-sericin solid dispersion for improved solubility and dissolution, N.H. Salunkhe et al./ International Journal of Biological Macromolecules xxx (2017) xxx-xxx,

7. PranaliWaghmare, Dr, PramodKadu, Solubility Enhancement of Curcumin Using HPMC K4M and HPMC K 15M By Solvent Change Precipitation Method,Vol 3, Issue 11, 2014,

8. A. Choudhary, A.C. Rana, G. Aggarwal, V. Kumar, F. Zakir, Development and characterization of an atorvastatin solid dispersion formulation usingskimmed milk for improved oral bioavailability, Acta Pharm. Sin. B 2 (2012)421-428,.

9. NantanaSittichai, sirichaiKrabesri, EkamolSuthisonAndParkpoamTengamnuay, an approach to developing dissolution standard's for turmeric capsule: basket rotating method, Thai J. Pharm. Sci. 31 (2007)83-90.

10. AchmadRadjiaram, AchmadFuad, DwiSetyawan, Dissolution Enhancement of Curcumin by Hydroxypropyl-B-CyclodextrinComplexation, Int J PharmaSci, Vol 5, Suppl 3, 401405.

11. ShardaSambhakar, Bishamber Singh, KirticaMadan, Monalisha, NehaKashyap, ShaliniMayle, Solid Dispersion: A Tool For Improving the Solubility and Dissolution of Metronidazole, Sambhakar et al. International Journal of Drug Delivery 5 (1) 94-98,

12. Chao Wu, Zhongyan Wang, ZhuangzhiZhi, Tongying Jiang, Jinghai Zhang, Siling Wang, Development of Biodegradable Porous Starch Foam For Improving Oral Delivery of Poorly Water Soluble Drugs, c.wu et al./International Journal of Pharmaceutics 403 (2011)162-169.

13. Iswarya Sridhar, AbhaDoshi, Bhagyashri Joshi, VandanaWankhede, JesalDoshi, Solid Dispersion : an Approach to Enhance Solubility of poorly Water Soluble Drug, Journal of Scientific and Innovative Research 2013;2(3):685-694.

14. ModiKushal, ModiMonali, Mishra durgavati, Panchal Mittal, SorathiyaUmesh. ShelatPragna, Oral Controlled release Drug Delivery System, ModiKushal et al. Int.Res, J. Pharm. 2013, 4(3). 